\title{
Association between the use of a stylet in endotracheal intubation and postoperative arytenoid dislocation: a case-control study
}

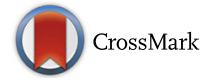

\author{
Lingeer $\mathrm{Wu}^{1}$, Le Shen ${ }^{1 *} \mathbb{D}$, Yuelun Zhang ${ }^{2}$, Xiuhua Zhang ${ }^{1}$ and Yuguang Huang ${ }^{1 *}$
}

\begin{abstract}
Backgrounds: Arytenoid dislocation (AD) is a rare but severe complication after general anesthesia with endotracheal intubation. We conducted a case-control study at Peking Union Medical College Hospital to identify risk factors associated with $A D$, including the use of an intubation stylet.

Methods: Patients who experienced AD were matched 1:3 with controls based on gender, age and type of surgery. Multiple conditional logistic regression was performed to determine associations between potential risk factors and AD.

Results: Twenty-six AD cases were retrospectively identified from 2004 through 2016. On average, arytenoid dislocation occurred in 2 cases per year, with an incidence of 0.904/100,000 (approximately 0.01\%). The 26 patients who experienced $A D$ and 78 matched control patients were enrolled in this study. All enrolled patients underwent endotracheal intubation, and a stylet was used for intubation for 38.5\% (10/26) of the AD patients and $64.1 \%$ (50/78) of the controls $(O R=0.23,0.07-0.74)$. A higher incidence of $A D$ was significantly associated with longer duration of operation $(\mathrm{OR}=1.74,1.23-2.47)$.

Conclusions: The use of an intubation stylet for endotracheal intubation appears to protect against AD. Prolonged operation time increases the risk of AD. These factors should be considered when assessing the risks of AD associated with endotracheal intubation and in efforts to avoid this complication.
\end{abstract}

Keywords: Arytenoid dislocation, Intubation stylet, Duration of operation, Case-control study, Postoperative complication

\section{Background}

Arytenoid dislocation (AD) is a rare postoperative complication after general anesthesia with tracheal intubation [1]. The incidence of $\mathrm{AD}$ after general anesthesia has been reported to be approximately 0 . $01-0.1 \%$ [2]. The cause of AD may be inadvertent trauma to the cricoarytenoid joint from the insertion of airway tools into the larynx [3-5]. To our knowledge, no systematic investigations of $\mathrm{AD}$ associated with tracheal intubation have been reported. Here, we report a hospital-based, case-control study to identify risk factors for surgery-associated AD.

\footnotetext{
* Correspondence: pumchshenle@aliyun.com; garypumch@163.com ${ }^{1}$ Department of Anesthesiology, Peking Union Medical College Hospital, Chinese Academy of Medical Sciences and Peking Union Medical College, Beijing, China

Full list of author information is available at the end of the article
}

\section{Methods}

This investigation was a retrospective hospital-based, case-control study approved by the Peking Union Medical College (PUMC) Hospital Institutional Review Board. The study protocol was designated S-K260 and was approved on May 11th, 2017. All the data were collected from the adverse-event system in Department of Anesthesiology. No written informed consent was obtained from the participants. A verbal consent to participation in this study of each participant was obtained through a phone call during follow-up, as all the participants had been discharged when we collected the data.

\section{Patients}

From January 2004 to December 2016, 26 cases involving postoperative $\mathrm{AD}$ were reported in the adverse-event system. Patients were matched 1:3 (case:control without AD) based on gender, age and type of surgery. We also 
recorded the following information for each patient: body mass index (BMI), surgical history, pre-existing laryngeal disease, comorbidities, smoking and alcohol history, American Society of Anesthesiologists physical status classification, number of intubation attempts, Cormack Lehane grading during intubation, intubating tools, size of the endotracheal tube, duration of operation, postoperative admission to the intensive care unit, whether a stylet was used to assist with intubation, and preoperative baseline laboratory data.

\section{Diagnosis of arytenoid dislocation}

All the patients with AD appear some similar symptoms within one week after the operations, such as, hoarseness, choking when drinking water, and cough. Then electronic fibro-laryngoscopy would be performed by attending otolaryngologists to make the final diagnostic reports.

\section{Statistical analysis}

Categorical variables were expressed as frequencies and percentages, which were compared using chi-squared tests. Continuous variables were summarized as means $\pm \mathrm{SD}$ and compared using Student's unpaired t-test. We use Bonferroni correction on the top of simple multiple t-tests to adjust the statistical significant level. Multiple conditional logistic regression was performed to determine associations between potential risk factors and AD. Statistical analyses were conducted using SPSS 19.0 (SPSS Inc., Chicago, IL, USA).

\section{Results}

A total of 287,767 cases involving surgery under general anesthesia were recorded at our hospital from 2004 to 2016, with an average of 22,136 such cases per year (Table 1). Twenty-six cases involving AD, an average of 2 such cases per year, were recorded; thus, the incidence of this complication was approximately $0.01 \%(0.904 / 100,000)$.

Table 2 lists basic information for the patients who experienced postoperative AD. These 26 patients included 14 males. Their ages ranged from 23 to 83 $(54.0 \pm 17.376)$ years. With respect to American Society of Anesthesiologists physical status classification, 8 patients, 15 patients, 2 patients, and 1 patient were categorized as class I, II, III, and IV, respectively. The types of surgery performed included various extensive procedures. In PUMC hospital, Macintosh direct laryngoscope is the primarily routine choice for intubation, and the endotracheal tube size is commonly ID 7.5 for male patients and ID 7.0 for female patients. All AD patients underwent tracheal intubation, and among them only one patient received 3 attempts of intubation with Shikani optical stylet. Three $\mathrm{AD}$ patients received a double-lumen bronchial tubes, the rest $23 \mathrm{AD}$ patients received single-lumen endotracheal tubes, with none of them received a laryngeal mask airway. $\mathrm{AD}$ occurred on the left side in 15 patients and on the right side in 11 patients. No patients in the both group suffered pre-existing laryngeal disease or arthritis before the operation. All AD patients recovered or improved after reduction procedure.

Table 3 presents comparisons between the cases and controls for numerous variables. The following differences were statistically significant. Relative to the controls, the $\mathrm{AD}$ patients had longer durations of operation and longer postoperative hospital stays, but less frequently underwent intubation involving the use

Table 1 Incidence of AD at the PUMCH from 2004 through 2016

\begin{tabular}{llll}
\hline Year & Total Cases of General Anesthesia & Total Cases of AD & Incidence of AD' (per 100,000) \\
\hline 2004 & 13,160 & 1 & 0.760 \\
2005 & 14,145 & 1 & 0.707 \\
2006 & 14,666 & 0 & 0.000 \\
2007 & 15,054 & 0 & 0.000 \\
2008 & 15,731 & 0 & 0.000 \\
2009 & 19,190 & 0 & 0.000 \\
2010 & 20,941 & 2 & 0.955 \\
2011 & 21,687 & 5 & 2.306 \\
2012 & 24,960 & 5 & 2.003 \\
2013 & 28,525 & 3 & 1.052 \\
2014 & 31,857 & 3 & 0.942 \\
2015 & 33,192 & 4 & 1.205 \\
2016 & 34,659 & 2 & 0.577 \\
Average Cases/Year & 22,136 & 2 & 0.904 \\
Total Cases & 287,767 & 26 & 0.904 \\
\hline
\end{tabular}


Table 2 Basic Information of Patients with Intraoperative AD

\begin{tabular}{|c|c|c|c|c|c|}
\hline Case ID & Gender & Age & ASA & Name of Surgery & AD side \\
\hline $\mathrm{A} 1$ & 2 & $50-59$ & $\|$ & Combined liver resection & L \\
\hline$A 2$ & 2 & $40-49$ & I & Exploratory laparotomy, Phemister & $\mathrm{R}$ \\
\hline A3 & 2 & $50-59$ & 1 & Radical operation for stomach carcinoma & $\mathrm{R}$ \\
\hline A4 & 1 & $70-79$ & I & Bowel resection & L \\
\hline A5 & 1 & $30-39$ & III & Vaginal stump bleeding in suture technique & L \\
\hline A6 & 2 & $20-29$ & IV & Exploratory laparotomy & L \\
\hline A7 & 2 & $20-29$ & I & Intracranial brain electrodes & L \\
\hline A8 & 2 & $80-89$ & I & Open cholecystectomy & $\mathrm{R}$ \\
\hline A9 & 1 & $60-69$ & $\|$ & Anterior approach decompression & L \\
\hline $\mathrm{A} 10$ & 1 & $20-29$ & $\|$ & Exploratory laparotomy & $\mathrm{R}$ \\
\hline A11 & 1 & $50-59$ & 1 & Hepatic lateral lobectomy & L \\
\hline $\mathrm{A} 12$ & 1 & $70-79$ & $\|$ & Segmental hepatectomy & L \\
\hline A13 & 2 & $40-49$ & I & Open thoracic exploration, aortic replacement & $\mathrm{R}$ \\
\hline A14 & 1 & $70-79$ & $\|$ & Whipple & $\mathrm{R}$ \\
\hline A15 & 1 & $60-69$ & III & CABG & L \\
\hline A 16 & 2 & $30-39$ & $\|$ & Aortic replacement & R \\
\hline $\mathrm{A} 17$ & 2 & $60-69$ & $\|$ & Miles & L \\
\hline $\mathrm{A} 18$ & 2 & $30-39$ & $\|$ & Aortic replacement & $\mathrm{R}$ \\
\hline A19 & 1 & $70-79$ & $\|$ & Total knee arthroplasty & L \\
\hline $\mathrm{A} 20$ & 2 & $60-69$ & $\|$ & Anterior approach decompression & R \\
\hline A21 & 1 & $70-79$ & $\|$ & LVATS & L \\
\hline A22 & 2 & $40-49$ & 1 & Whipple & L \\
\hline A23 & 2 & $60-69$ & $\|$ & LVATS & L \\
\hline A24 & 2 & $50-59$ & $\|$ & Radical operation for cardia carcinoma & $\mathrm{R}$ \\
\hline A25 & 1 & $60-69$ & $\|$ & Retroperitoneal neoplasm resection & L \\
\hline A26 & 2 & $40-49$ & $\|$ & Exploratory laparotomy & L \\
\hline
\end{tabular}

of an intubation stylet. The duration of operation was $4.03 \pm 1.67 \mathrm{~h}$ for the cases and $2.60 \pm 1.52 \mathrm{~h}$ for the controls $(P<0.002)$. The two groups did not significantly differ with respect to age; gender; BMI; depth of intubation; frequency of postoperative admission to the intensive care unit; rates of hypertension, diabetes mellitus, smoking, or alcohol use; or results for several preoperative laboratory tests, including assessments of serum albumin concentration, alanine aminotransferase, prothrombin time, and activated partial thromboplastin time.

Table 4 illustrates results from a multiple logistic regression model used to quantify associations between potential risk factors and AD. Intubation with a stylet was associated with a significantly reduced risk of surgery-associated AD (OR $=0.23,0.07-0.74, P<0.01$ ), and longer duration of operation was associated with a higher risk of $\mathrm{AD}(\mathrm{OR}=1.89$, $1.31-2.74, \mathrm{P}<0.01)$. Frequency of postoperative admission to the intensive care unit, American Society of Anesthesiologists class III-IV status, BMI, and serum albumin concentration were not significant risk factors.

\section{Discussion}

$\mathrm{AD}$ is a complication that is known to occur in all surgical departments, and factors that may either cause or help to prevent AD should be considered. Our incidence rate of $\mathrm{AD}$ (approximately $0.01 \%$ ) is consistent with rates reported by Szigeti et al. [1] and other researchers [2]. The primary finding of our study is that the use of an intubation stylet was a significant protective factor for AD. Although certain authors have reported possible disadvantages of stylet use, including an increased incidence of postoperative pharyngeal pain [6] and an increased frequency of sore throat [7] caused by removal of the stylet. As a teaching hospital, most intubation attempts were conducted by residents under 5 years, or even medical interns, unless the intubation is too difficult. So intubation with a stylet is strongly encouraged especially for those trainees. The reason might be that stylet could decrease violence of laryngeal scope during exposing the glottis. An incidental but noteworthy finding of our study is that all of the patients in the case group 
Table 3 Comparsion between Case and Control

\begin{tabular}{|c|c|c|c|}
\hline Variables & Case $(n=26)$ & Control $(n=78)$ & $P$ value \\
\hline Age $($ mean \pm std) & $54 \pm 17.37$ & $54 \pm 17.37$ & 1.000 \\
\hline Male, N (\%) & $15(57.7)$ & $45(57.7)$ & 1.000 \\
\hline BMI (mean \pm std) & $23.50 \pm 3.69$ & $23.19 \pm 3.35$ & 0.6863 \\
\hline Length of Surgery (mean \pm std) & $4.03 \pm 1.66$ & $2.60 \pm 1.51$ & 0.0002 \\
\hline Depth of Intubation (mean \pm std) & $23.00 \pm 1.85$ & $22.60 \pm 1.77$ & 0.1366 \\
\hline Number of attempts > 1,n (\%) & $1(3.85)$ & $3(3.85)$ & 1.000 \\
\hline Cormack Lehane Grade I, n (\%) & $18(69.2)$ & $60(76.9)$ & 0.438 \\
\hline Postopertive Hospital Days (mean \pm std) & $27.64 \pm 12.16$ & $15.88 \pm 9.71$ & $<0.0001$ \\
\hline Past Surgical History, n (\%) & $8(30.76)$ & $23(29.48)$ & 1.000 \\
\hline Hypertension, n (\%) & $6(23.07)$ & $25(32.05)$ & 0.464 \\
\hline Diabetes, n (\%) & $4(15.38)$ & $12(15.38)$ & 1.000 \\
\hline Somking, n (\%) & $5(19.23)$ & $23(29.48)$ & 0.444 \\
\hline Alcohol Drinking, n (\%) & $5(19.23)$ & $14(17.95)$ & 1.000 \\
\hline Postoperative ICU Admit, n (\%) & $5(19.23)$ & $10(12.82)$ & 0.519 \\
\hline Stylet Assisted Intubation, n (\%) & $10(38.46)$ & $50(64.10)$ & 0.038 \\
\hline${ }^{a}$ ASA I-II, n (\%) & $23(88.46)$ & $63(80.76)$ & 0.551 \\
\hline \multicolumn{4}{|l|}{ Complete Cell Count } \\
\hline $\operatorname{RBC}\left(10^{12} / \mathrm{L}\right)$, mean \pm std & $4.08 \pm 0.739$ & $4.38 \pm 0.533$ & 0.0268 \\
\hline WBC $\left(10^{9} / L\right)$, mean \pm std & $7.73 \pm 2.98$ & $6.503 \pm 2.903$ & 0.0127 \\
\hline Hemoglubin $(\mathrm{g} / \mathrm{L})$, mean \pm std & $123.81 \pm 22.53$ & $132.71 \pm 19.74$ & 0.0516 \\
\hline Platelet $\left(10^{9} / \mathrm{L}\right)$, mean \pm std & $216.60 \pm 82.60$ & $221.62 \pm 83.34$ & 0.5845 \\
\hline Albumin $(\mathrm{g} / \mathrm{L})$, mean $\pm \mathrm{std}$ & $38.96 \pm 5.72$ & $40.73 \pm 4.97$ & 0.0911 \\
\hline $\operatorname{ALT}(U / L)$, mean $\pm s t d$ & $75.23 \pm 196.22$ & $22.69 \pm 31.29$ & 0.2161 \\
\hline PT $(s)$, mean \pm std & $12.81 \pm 3.72$ & $12.15 \pm 1.361$ & 0.9263 \\
\hline $\operatorname{APTT}(s)$, mean \pm std & $30.25 \pm 11.45$ & $28.46 \pm 6.241$ & 0.7073 \\
\hline
\end{tabular}

a: American Society of Anesthesiologists

Table 4 Risk Assessment of Intraopeartive AD

\begin{tabular}{llll}
\hline Variables & Unadjusted OR & $95 \%{ }^{\mathrm{a} C l}$ & $P$ value \\
Use of Stylet & 0.35 & $0.14-0.87$ & 0.03 \\
Duration of Operation & 1.71 & $1.26-2.33$ & 0.00 \\
ASA & 1.08 & $0.58-2.00$ & 0.81 \\
BMI & 1.03 & $0.90-1.17$ & 0.69 \\
Tube Depth & 1.10 & $0.88-1.38$ & 0.39 \\
Variables & adjusted OR & $95 \%{ }^{\mathrm{a} C l}$ & $P$ value \\
Use of Stylet & 0.23 & $0.07-0.74$ & 0.01 \\
Duration of Operation & 1.89 & $1.31-2.74$ & 0.00 \\
ASA & 1.05 & $0.90-1.23$ & 0.51 \\
BMI & 1.01 & $0.48-2.11$ & 0.99 \\
Tube Depth & 1.23 & $0.94-1.62$ & 0.14 \\
\hline
\end{tabular}

a: confidence interval underwent intubation; none of these patients received laryngeal masks. Therefore, the use of a laryngeal mask could be an approach for avoiding the occurrence of postoperative $\mathrm{AD}$. In addition, we found that the severity of a patient's health status (specifically, being ASA class III-IV) and BMI were not related to the incidence of AD.

Another important finding of our study was that duration of operation was significantly longer for the $A D$ patients than for the control patients. Logistic analysis showed that no stylet use and length of surgery were independent risk factors for AD. Other authors [8] have reported that prolonged duration of anesthesia was a significant risk factor for the occurrence of AD and suggested that pressure exerted by a tracheal tube is a cause of AD [8]. However, it is difficult to separate the effects of stylet use and length of surgery. Another of our observations was that hospital stay was significantly longer for the patients with $\mathrm{AD}$ than for the patients without $\mathrm{AD}$; we suspect that this difference is related to complexity of the surgical procedure but not to stylet use. 
We also found that compared with the control group, the case group had significantly lower preoperative peripheral red blood cell counts and significantly higher white blood cell counts; however, the blood counts of both groups were within the normal range, and logistic analysis showed that neither of these counts was an independent risk factor for $\mathrm{AD}$. Therefore, the significance of these hematologic findings with respect to $A D$ is unclear. Anemia or joint inflammation may lead to instability [9], but it is not known whether either of these phenomena is relevant to the cause of AD. The results of several other common laboratory tests were also not significantly associated with $\mathrm{AD}$; thus, our study did not identify a preoperative laboratory test that could predict the occurrence of AD.

In our series, no cases of $\mathrm{AD}$ were recorded during 2006-2009. We suspect that the reason for this phenomenon is that the Department of Anesthesiology's adverse-event system was improved after 2010, with better follow-up of anesthesiologists' performance. So there should be some missing $\mathrm{AD}$ cases in the cohort, as is a major limitation for our research. So we need to improve our adverse event reporting system to achieve more cases in future.

This study has some other limitations. For example, this study was a retrospective case-control study and may therefore have incorporated selection bias. Age, gender and surgery department could not be analyzed in this investigation. Secondly, since postoperative AD is a rare complication, our case group contained only 26 patients. Large, multi-center prospective trials may be needed to conclusively establish whether the use of an intubation stylet protects against AD.

In conclusion, $\mathrm{AD}$ is a rare but severe complication after general anesthesia. Use of an intubation stylet appears to protect against $\mathrm{AD}$, and longer duration of operation favors the occurrence of this complication. These factors should be considered when assessing the risks of $\mathrm{AD}$ associated with endotracheal intubation and in efforts to avoid this complication.

\section{Conclusion}

The use of an intubation stylet for endotracheal intubation appears to protect against AD. Prolonged operation time increases the risk of AD. These factors should be considered when assessing the risks of $\mathrm{AD}$ associated with endotracheal intubation and in efforts to avoid this complication.

Acknowledgements

We are grateful to Dr. Min Yao for valuable discussion.

\section{Availability of data and material}

The datasets generated and analyzed during the current study are available from the corresponding author on reasonable request.
Funding

There's no funding in this manuscript.

\section{Authors' contributions}

LW collected the data, conduct statistic analysis and drafted the manuscript. LS directed the design of the study, modify the article. YZ participated in the statistical analysis. XZ participated in the quality control of the study. YH conceived of the study, and participated in its design and coordination. All authors read and approved the final manuscript.

\section{Ethics approval and consent to participate}

This investigation was a retrospective hospital-based, case-control study approved by the Peking Union Medical College (PUMC) Hospital Institutional Review Board. All the data were collected from the adverse-event system in Department of Anesthesiology. No written informed consent was obtained from the participants. A verbal consent to participation in this study of each participant was obtained through a phone call during follow-up, as all the participants had been discharged when we collected the data. The study protocol was designated S-K260 and was approved on May 11th, 2017.

Consent for publication

I declare to consent for publication and there's no individual identifying data.

Competing interests

The authors declare that they have no competing interests.

\section{Publisher's Note}

Springer Nature remains neutral with regard to jurisdictional claims in published maps and institutional affiliations.

\section{Author details}

'Department of Anesthesiology, Peking Union Medical College Hospital, Chinese Academy of Medical Sciences and Peking Union Medical College, Beijing, China. ${ }^{2}$ Central Research Laboratory, Peking Union Medical College Hospital, Chinese Academy of Medical Sciences and Peking Union Medical College, Beijing, China.

Received: 18 February 2018 Accepted: 11 May 2018

Published online: 31 May 2018

\section{References}

1. Szigeti CL, Baeuerle JJ, Mongan PD. Arytenoid dislocation with lighted stylet intubation: case report and retrospective review. Anesth Analg. 1994;78(1): 185-6.

2. Tan PH, Hung KC, Hsieh SW, Chen TB, Liu PH, Chen WH. Large-bore calibrating orogastric tube and arytenoid dislocation: a retrospective study. $\mathrm{Br} J$ Anaesth. 2016;1 16(2):296-8

3. Hung KC. Arytenoid cartilage dislocation after laparoscopic surgery for treatment of diabetes. A \& A case reports. 2013;1 (2):34-6.

4. Kakushima N, Hotta K, Tanaka M, Kawata N, Sawai H, Imai K, Takao T, Takao M, Takizawa K, Matsubayashi H, et al. Anterior arytenoid cartilage dislocation, a rare complication of esophagogastroduodenoscopy. Endoscopy. 2012;44(Suppl 2 UCTN):E363.

5. Niwa Y, Nakae A, Ogawa M, Takashina M, Hagihira S, Ueyama H, Mashimo T. Arytenoid dislocation after cardiac surgery. Acta Anaesthesiol Scand. 2007; 51(10):1397-400.

6. Komasawa N, Nishihara I, Minami T. Effects of stylet use during tracheal intubation on postoperative pharyngeal pain in anesthetized patients: a prospective randomized controlled trial. J Clin Anesth. 2017:38:68-70.

7. Kusunoki T, Sawai T, Komasawa N, Shimoyama Y, Minami T. Correlation between extraction force during tracheal intubation stylet removal and postoperative sore throat. J Clin Anesth. 2016;33:37-40.

8. Yamanaka H, Hayashi $Y$, Watanabe $Y$, Uematu H, Mashimo T. Prolonged hoarseness and arytenoid cartilage dislocation after tracheal intubation. $\mathrm{Br} \mathrm{J}$ Anaesth. 2009;103(3):452-5.

9. Rieger A, Hass I, Gross M, Gramm HJ, Eyrich K. Intubation trauma of the larynx-a literature review with special reference to arytenoid cartilage dislocation. Anasthesiologie, Intensivmedizin, Notfallmedizin, Schmerztherapie : AINS. 1996;31(5):281-7. 